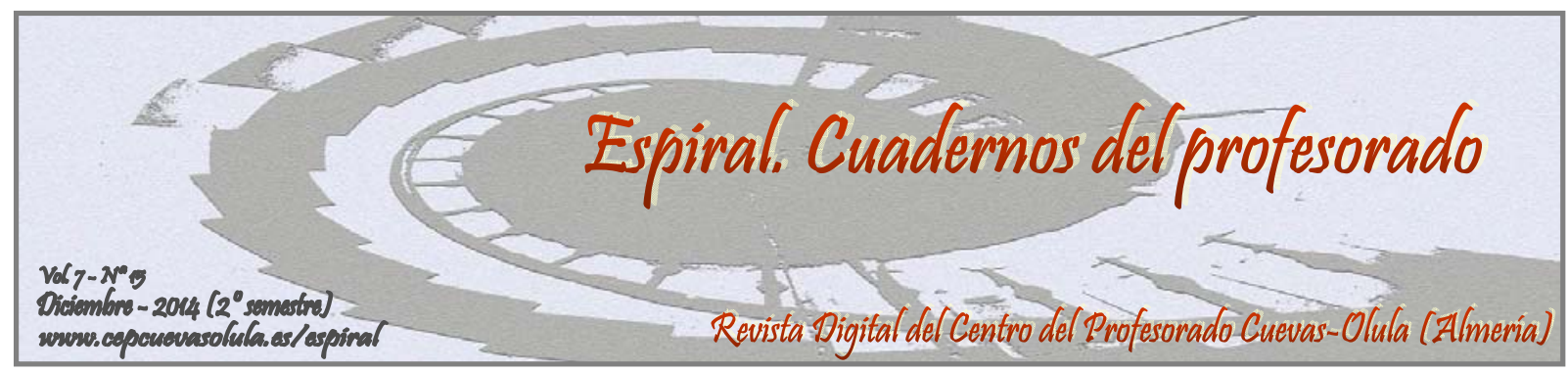

\title{
PROPUESTA PARA LA ORGANIZACIÓN DE UN TRIATLÓN CROSS EN EL TÉRMINO MUNICIPAL DE LORCA
}

\section{PROPOSAL FOR THE ORGANIZATION OF AN OFF-ROAD TRIATHLON IN THE TOWN OF LORCA}

\section{Adrián Fernández Hernández y Patricio Palao Puche}

Universidad de Murcia, España

\begin{abstract}
RESUMEN: Gracias a la ubicación geográfica de la ciudad de Lorca y por su tradición deportiva se darán una serie de claves para la celebración y organización de un triatlón cross dentro de su territorio municipal detallando así: circuito de natación, circuito de bicicleta de montaña, circuito de carrera a pie, transiciones, avituallamientos, categorías, así como el material necesario para llevarlo a cabo. Dicha propuesta se encuentra sustentada en el amplio medio natural, la climatología, la afición popular y el patrimonio cultural de la ciudad estableciendo un límite de participantes en vista a la preservación del entorno natural mediante la imposición de normas y el establecimiento de zonas especiales para la deposición de los desechos por parte de los participantes. Por último, se plantean una serie de proyectos de cara al futuro si esta propuesta acabara, finalmente, exitosa ya no sólo en lo que se refiere a mejoras en la propia carrera sino al posible despliegue de todo un conjunto de pruebas de este tipo a nivel regional.
\end{abstract}

Palabras clave: medio natural, recorridos, transiciones, avituallamientos, conservación.

ABSTRACT: Due to the geographic location of the town of Lorca and because of its tradition in sports a kind of keys will be given to celebrate and organize an off-road triathlon detailing: the swimming sector, mountain-bike sector, running sector, transitions, provisioning points, categories and the material needs to make it. This proposal is based on the large natural environment, the weather, the supporters, the cultural patrimony of the city, fixing a limited number of participants regarding to the environment preservation by making rules and establishing special areas where every participant could trough away their wastes. In conclusion, it has been considerate the possibility of promote different off-road triathlons at regional level if this proposal could finally succeed.

Key words: natural environment, sectors, transitions, provisioning points, preservation.

Fernández Hernández, A. y Palao Puche, P. (2014). Propuesta para la organización de un triatlón cross en el término municipal de Lorca. Espiral. Cuadernos del Profesorado, 7(15), 64-69. Disponible en: http://www.cepcuevasolula.es/espiral

Fecha de recepción: 11/03/2014

Fecha de aceptación: 30/06/2014
Enviar correspondencia a: adrian.lorca94@gmail.com

\section{1.- INTRODUCCIÓN}

La mayoría de las pruebas disputadas tanto en España como en la Región de Murcia, que engloban al triatlón, están organizadas dentro de entornos urbanos. Existe una modalidad de este deporte llamada triatlón cross, la cual se desarrolla dentro de enclaves naturales. Consideramos así, 
que existe una gran carencia en cuanto a la celebración de este tipo de eventos. Por ello, en este trabajo se sugiere la posibilidad de organizar una prueba de estas características dentro del medio natural del término municipal de Lorca. El porqué de la realización de este triatlón cross en esta zona y no en otra, se fundamenta en las posibilidades que nos ofrece este espacio geográfico, el cual nos permite desarrollar los tres sectores deportivos que comprenden este deporte, disfrutando de la belleza del paisaje por el que discurre el recorrido. Además, debido al el elevado número de licencias federativas tanto a nivel nacional como regional y la gran participación de estos deportistas en todo tipo de pruebas de triatlón, este evento tendría una gran acogida por parte del público federado. Por otro lado, se destaca el compromiso deportivo de esta ciudad, que fue nombrada en el año 2013 Ciudad Europea del Deporte. Dicha prueba despertaría un gran interés en la población lorquina, dando a conocer este deporte cada vez más consolidado y mostrando así las posibilidades que nos ofrece la naturaleza siempre y cuando se respete.

Con respecto a el triatlón como deporte, autores como Le Breton (2009) lo han encasillado dentro de los deportes relacionados con el medio natural, en lo que coincidimos, pero queremos matizar esta perspectiva planteando el deporte del triatlón y en concreto, la modalidad de triatlón cross como la máxima expresión y definición de este deporte como actividad física en el medio natural. Se ha seguido la referencia que plantea Machota (2012), la cual toma como punto de partida la definición de actividad en el medio natural aportada por Tierra (1996) y, por otro lado, la clasificación de las actividades en la naturaleza según el entorno implicado para su desarrollo de Granero (2007), en la cual se clasifican las actividades en la naturaleza como: actividades de tierra, aire, agua y del mundo animal. Machota (2012) recoge esta clasificación para justificar el triatlón como actividad en el medio natural, siendo esta una actividad dentro de "actividades acuáticas y actividades de tierra cuando se realiza con una bicicleta de montaña y la carrera a pie se desarrolla en un entorno natural". Esta autora concreta el contexto en las modalidades de tierra pero no realiza ninguna matización sobre la natación. Por ello, a continuación especificaremos la clasificación de cada una de las disciplinas correspondientes a este deporte en su versión cross, pues para que un triatlón sea completamente una actividad en el medio natural, deben cumplirse los siguientes dos requisitos imprescindibles: el primero, la natación deberá darse en alguno de estos espacios naturales: mar, pantano, río o lago, y el segundo, que los sectores de ciclismo y carrera a pie deberán ser desarrollados por terrenos no urbanos y en todo momento en contacto con la naturaleza al igual que la bicicleta de montaña.

Siempre que se habla de cualquier evento deportivo de estas características tenemos que tener bien presente que hay que seguir un plan de sostenimiento del medio natural como el que nos plantean Luque, Baena y Granero (2011) y esta propuesta se haría respetando en todo momento los espacios naturales en los que se dispute la prueba.

El objetivo de este artículo es llevar a cabo una propuesta de triatlón cross en el término municipal de Lorca, dicha propuesta cumpliría todos estos requisitos establecidos.

\section{2.- MATERIAL Y MÉTODO}

La posible realización de este triatlón cross se realizaría en el territorio del Municipio de Lorca. Para esto, es importante delimitar el recorrido propuesto, qué es necesario para llevarlo a cabo y cómo se realizará:

\section{Circuito de natación}

El lugar planteado para el circuito de natación sería el Pantano de Puentes. Este pantano se localiza hacia el oeste de la ciudad de Lorca en dirección a las pedanías del oeste del municipio como son El Consejero o La Parroquia. En concreto, dicho pantano se encuentra situado en plena rambla del Río Guadalentín. Por su situación, el municipio de Lorca posee una gran variedad de parajes acuáticos. Se podría plantear, también, la realización de un circuito de natación en las playas naturales de Puntas de Calnegre, lo cual cambiaría la tipología de la prueba. Sin embargo, la ubicación del embalse de Puentes es idónea para realizar, en sí, una prueba de gran atractivo. Además, el factor clave que determina una prueba de este calibre es la capacidad de agua que posee el propio medio acuático. Es por esto, que con la capacidad actual se podría realizar sin problema alguno una prueba de este tipo al 
igual que en antecedentes anteriores (Tabla 1). Así, en el Raid de Aventura de Lorca que se realiza cada año en celebración de los Juegos Deportivos del Guadalentín se han llegado a realizar pruebas acuáticas como son natación o piragüismo.

Tabla 1. Capacidad Actual Pantano de Puentes (Fuente: embalses.net).

Embalse: PUENTES

\begin{tabular}{lrr}
\hline Agua embalsada (11-03-2014): & $10 \mathrm{hm}^{3}$ & $38.46 \%$ \\
\hline Variacion semana Anterior: & $0 \mathrm{hm}^{3}$ & $0.00 \%$ \\
\hline Capacidad: & $26 \mathrm{hm}^{3}$ & \\
\hline Misma Semana (2013): & $15 \mathrm{hm}^{3}$ & $57.69 \%$ \\
\hline Misma Semana (Media 10 Años): & $4 \mathrm{hm}^{3}$ & $17.69 \%$
\end{tabular}

La prueba de natación consiste en un circuito aproximado de entre 1200 y 1500 metros, para ello se realizarían dos vueltas al recorrido el cual estaría marcado con tres boyas de referencia formando un triángulo que los participantes deberían dejar a la izquierda. Dicho recorrido estaría también marcado y controlado por tres piragüistas que actuarían ante posibles rescates. La salida podría realizarse tanto dentro como fuera del agua. Además, las vueltas a dicho circuito serían también saliendo del agua o por dentro de la misma. Si la vuelta se realiza por dentro la boya de referencia más cercana a la línea de salida se debería dejar a la izquierda girando $180^{\circ}$ comenzando de nuevo el circuito. Si la vuelta se realiza por fuera del agua se colocaría una señalización visible la cual se tomaría igual que la boya de referencia con la diferencia de que esta se debería pasar a pie. Para controlar el número de vueltas de cada participante, aquellos que lleven una vuelta deberán pasar por la boya de referencia más cercana a la línea de salida. En cambio, para salir del agua hacia la Transición 1 se colocaría una calle de salida con corcheras escorada del circuito por donde se deberá salir hacia boxes (Figura 1).

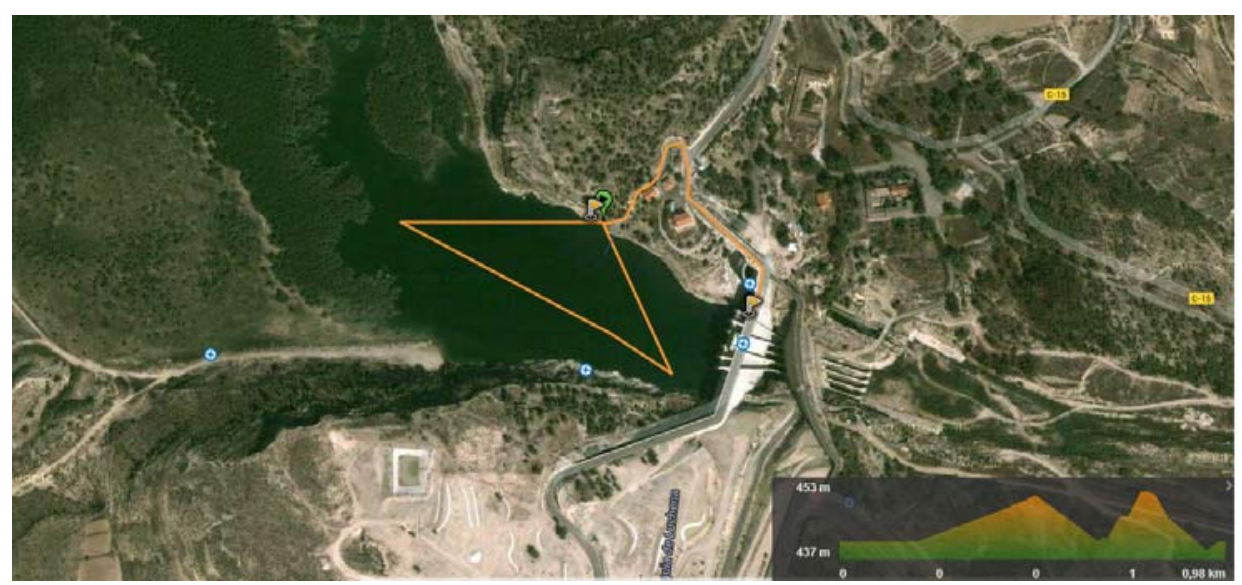

Figura 1. Circuito Natación y Calle de Salida hasta T1 (Fuente: wikiloc.com)

\section{Circuito en bicicleta}

Una vez la natación se ha acabado comienza el tramo de bicicleta de montaña o mountain bike, se trataría de un recorrido de alrededor de unos 32 kilómetros, de un solo sentido, donde se mezclan distintos tipos de terreno. El circuito comenzaría en dirección Lorca bajando por la rambla del Río Guadalentín, y a la altura de El Consejero se tomaría un cruce que introduce al participante en plena Sierra de la Peñarrubia lorquina pasando por parajes tan conocidos como la Rambla de los 17 arcos o la Finca del Praico (Figura 2). Conforme los kilómetros van pasando se aumenta el desnivel positivo del recorrido lo cual aumenta la dificultad de la prueba. La altura máxima a la que se llegaría en el circuito de montain bike es de $766 \mathrm{~m}$ de altitud (Figura 3). La dificultad en cuanto a aspectos organizativos de este recorrido residiría en la coordinación de las fuerzas de seguridad controlando el 
tráfico en los primeros kilómetros sobre todo, los servicios de emergencia que cubrirían la prueba al completo y los voluntarios que guiarían a los participantes en moto junto con las señalizaciones establecidas.

\section{Circuito de carrera a pie}

Tras la prueba de ciclismo comenzaría la carrera a pie en forma de recorrido de trail de montaña. Se trataría de 10 kilómetros a pie en los cuales se empezaría en la última parte de ascensión hacia el pico de la Peñarrubia y se descendería hacia el itinerario turístico del Cejo de los Enamorados. Posteriormente, se iría hacia la rambla de la Viña en concreto a la parte conocida como "El Recodo" donde se enlazaría con la meta en el Castillo de Lorca (Figura 4). Es pues, un recorrido complicado y de exigencia física, pero que se encuentra marcado en todo momento pues es en su mayoría parte de un itinerario ya establecido.

\section{Transiciones}

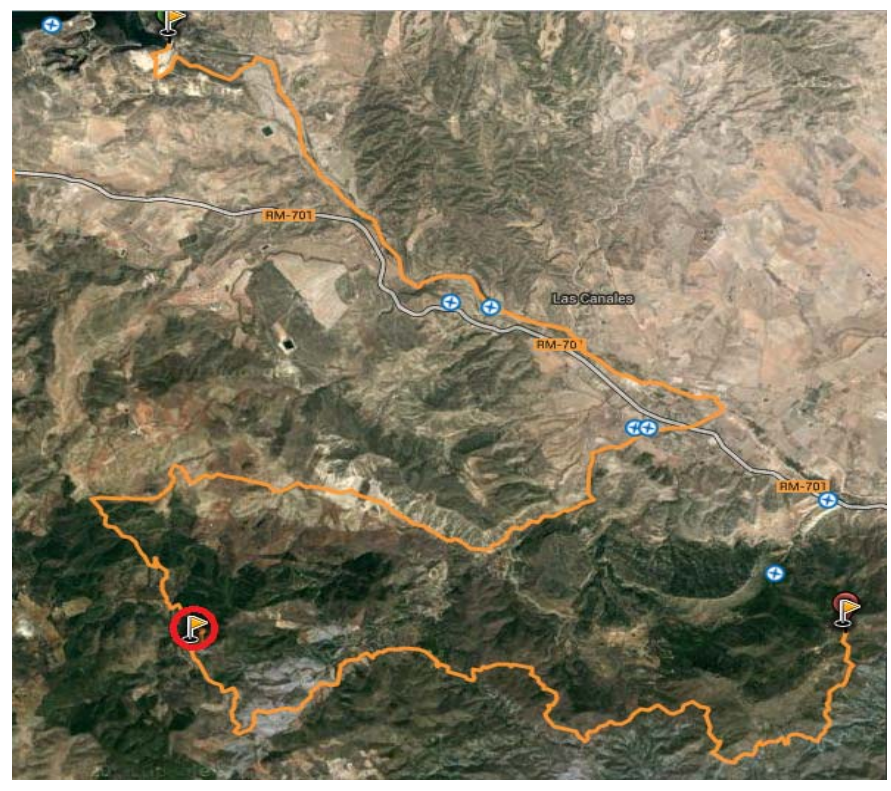

Figura 2. Circuito de bicicleta (Fuente: wikiloc.com)

La transición o box en un triatlón es el espacio adaptado en el cual los participantes realizan el cambio de una disciplina a otra. Las transiciones contienen una gran parte del reglamento en una competición de triatlón y se trata de recorridos neutros donde los circuitos comienzan tras la línea de salida de boxes. En este triatlón se utilizarían dos transiciones: Transición 1de natación a bicicleta y Transición 2-de bicicleta a

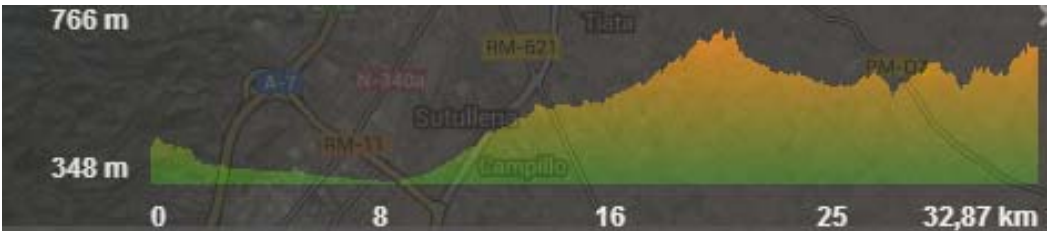

Figura 3. Perfil Circuito de bicicleta (Fuente: wikiloc.com) carrera a pie. Ambas transiciones tendrían las mismas características. Estarían formadas por barras de metal alargadas que sirven para apoyar las bicicletas. En cada tramo de medio metro o metro se irían colocando los distintos dorsales correspondientes a cada participante. Estos se colocarían de tal forma que fueran

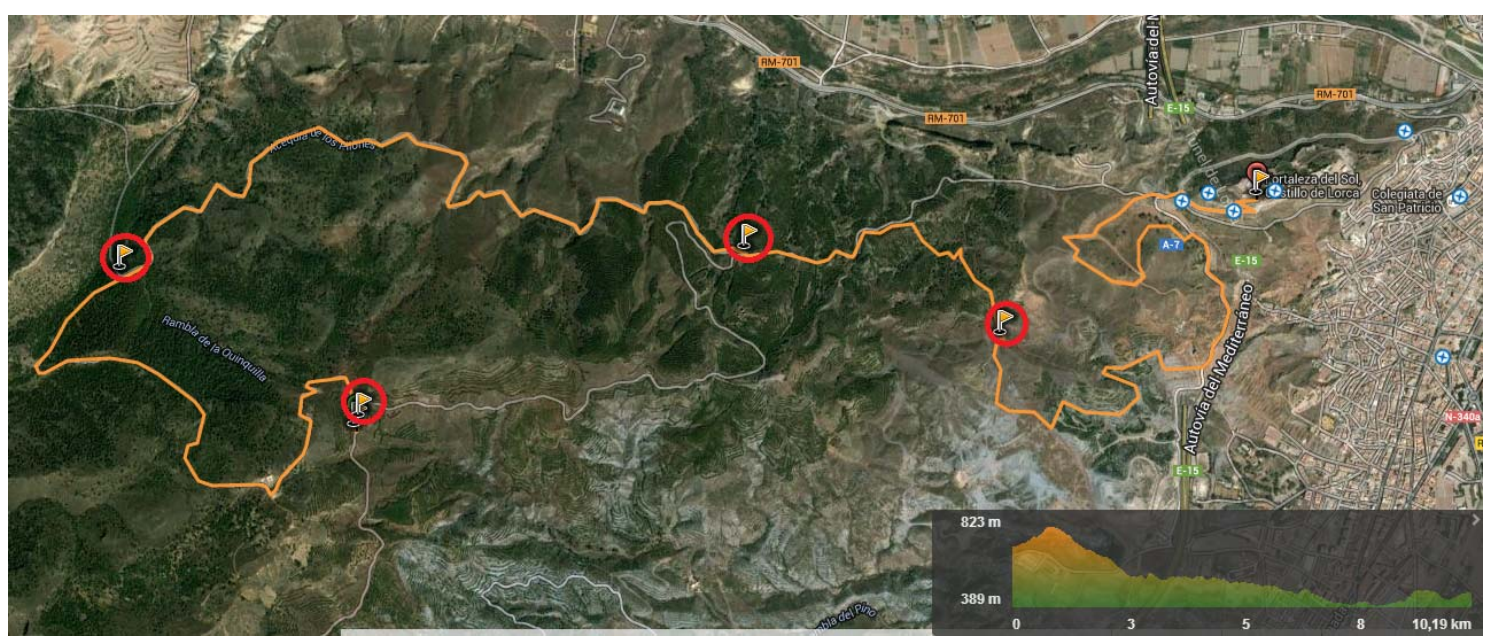

Figura 4. Circuito carrera a pie(Fuente: wikiloc.com) 
fácilmente visibles por estos. Bajo cada dorsal, en la Transición 1, se establecerían cestas donde los participantes depositarían los materiales utilizados en la natación. Una vez el último participante de natación haya salido del agua y comience el circuito ciclista los voluntarios recogerían los materiales en bolsas numeradas por dorsal que serían depositadas en meta para que cada participante pudiera recogerla. Lo mismo ocurriría en la Transición 2, donde debido a la delicadeza del material a recoger (bicicletas de montaña) el participante podría elegir recoger su material él mismo al término de la prueba o que la organización recoja el material mediante un transporte especialmente equipado para ello. Cada transición estaría aislada mediante vallas no permitiendo la entrada de público en ella y dónde solo se permitiría la presencia de los atletas y los jueces.

\section{Avituallamientos}

Los avituallamientos son una parte importante en la organización de la prueba pues el desgaste de la misma genera la necesidad en los participantes de ingerir líquidos para poder continuar en las mejores condiciones físicas posibles. En esta propuesta de triatlón cross se establecerían cinco avituallamientos a lo largo del recorrido total, en concreto habría un avituallamiento en el circuito ciclista (Figura 2) y cuatro avituallamientos en el recorrido a pie (Figura 4). Dichos avituallamientos estarían situados en zonas estratégicas que por la morfología llana y amplia del terreno en esas partes del circuito permitirían delimitar zonas donde los atletas podrían coger los líquidos y depositarlos dentro de unas zonas especialmente marcadas donde posteriormente unos voluntarios recogerían los desperdicios evitando así el impacto medioambiental que se podría causar.

\section{Categorías}

Las categorías establecidas para este triatlón irían en función del número de participantes que tomen salida el cual se establecería en un máximo de 250-300 personas. Las categorías como sugiere la compañía organizadora de triatlón cross, Xterra (2014), son: “Élite, A: 15 - 19; B: 20 - 24; C: 25 29; D: 30 - 34; E: 35 - 39; F: 40 - 44; G: 45 - 49; H: 50 - 54 I: 55 - 59; J: 60 - 64; K: 65 - 69; L: $70+"$.

\section{3.- CONCLUSIONES}

El hecho de plantear una prueba de esta categoría en un lugar como es el término municipal de Lorca se sustenta en firmes convicciones. Consideramos que este municipio agrupa una serie de condiciones que, en combinación, permiten llevar a cabo un triatlón cross. Dichas condiciones se concretan en: posesión de un amplio medio natural cercano a la ciudad con una orografía elevada, meteorología templada/calurosa a lo largo de todo el año por lo que los riesgos por suspensión de la prueba debido a causas meteorológicas se reducen ampliamente, afición popular por este deporte, y existencia de un gran patrimonio cultural durante el recorrido de la prueba.

Otro aspecto importante que se quiere destacar es el número de participantes limitado que se propone. Es evidente, que el desgaste medioambiental depende en gran medida de la cantidad de corredores que tomen salida en la prueba. Es por esto que se establecería un límite de participación de entre los 250 y los 300 participantes. Sería también necesario establecer zonas especiales en los avituallamientos donde se permitiría a los atletas desechar los distintos envoltorios que utilicen a lo largo de la prueba y que serían recogidos por los voluntarios inmediatamente después de su depósito en estas zonas. A cualquier corredor que arrojase sus deshechos fuera de estas zonas y fuera visto por jueces o voluntarios se le aplicaría una sanción que iría en relación al reglamento de la prueba.

Además, gracias al amplio territorio de Lorca, ante cualquier imprevisto por el cual no se pudiera llevar a cabo esta propuesta dentro del recorrido previsto se podría reaccionar cambiando parcialmente la ubicación de la prueba con antelación. En concreto, se realizaría el sector de natación en las playas de Puntas de Calnegre. La bicicleta de montaña se haría atravesando la sierra de Morata y enlazando con la ciudad de Lorca se podría acabar en la misma meta propuesta anteriormente en el Castillo de Lorca. En cambio, este tipo de modificaciones cambiaría por completo la tipología de la prueba en lo que se refiere a distancias sobre todo.

Por último, se cree necesario hacer referencia a futuros proyectos nacientes tras esta prueba. Si tuviera una buena acogida por parte de los participantes y el público se debería plantear un circuito a 
nivel regional de triatlón cross, pues actualmente el calendario tanto regional como nacional escasea en pruebas de este tipo. Los deportes, en el medio natural, son una propuesta en auge actualmente por su aspecto desafiante. La premisa principal al organizar este tipo de pruebas es la de ofrecer al participante una carrera exigente, que cumpla con las expectativas tanto a nivel personal del corredor como organizativo por parte del comité organizador. Es por esta razón, por la que sería conveniente llevar a cabo un planteamiento serio que consiga poner en comunión lo anteriormente citado. Por tanto, se debe ofrecer una prueba de calidad en todos los aspectos que incurren a la organización de una prueba deportiva, que busque mejorar progresivamente cada año y que contemple el cuidado del medio natural como la parte más importante a tener en cuenta.

\section{4.- REFERENCIAS}

Embalses.net. (2014). Embalse: Puentes (tiempo real). Dirección URL: http://www.embalses.net/pantano-816puentes.html [Consulta: 7 de abril 2014].

FETRI. (2014). Página web de la Federación Española de triatlón. Dirección URL: http://triatlon.org/triweb/ [Consulta: 7 Abril 2014].

Granero, A. (2007). Una aproximación conceptual y taxonómica a las actividades físicas en el medio natural. Lecturas: Educación Física y Deportes. http://www.efdeportes.com/ , 107.

Le Breton, D. (2009). Pasiones del riesgo y contacto con la naturaleza. Educación Física y Ciencia, 11, 13-31.

Luque, P. Baena, A. y Granero, A. (2011). Buenas prácticas para un desarrollo sostenible en los eventos deportivos en el medio natural. Interciencia: Revista de ciencia y tecnología de América, 36(7), 531537.

Machota, V. (2012). Propuesta de una unidad didáctica de triatlón en el marco escolar. EmásF: revista digital de educación física, 19, 115-129.

Tierra, J. (1996). Actividades recreativas en la naturaleza. En Machota, V. (2012). Propuesta de una unidad didáctica de triatlón en el marco escolar. EmásF: revista digital de educación física, 19, 115-129.

Wikiloc.com. (2014). Dirección URL: http://es.wikiloc.com/wikiloc/home.do[Consulta: 7 de abril 2014].

Xterra. (2014). Página web de la empresa Xterra. Dirección URL: http://xterraspain.com/ [Consulta: 8 Abril 2014].

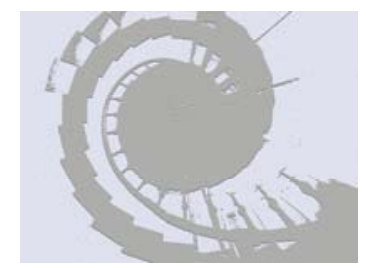

